\title{
Electromagnetic field manipulation in planar nanorod complexes metamaterial for slow light application
}

\author{
Junqiao Wang ${ }^{1}$, Jia Zhang ${ }^{1}$, Chunzhen Fan $^{1}$, Kaijun Mu ${ }^{1, *}$, Erjun Liang ${ }^{1}$, and Pei Ding ${ }^{2}$ \\ ${ }^{1}$ School of Physical Science and Engineering and Key Laboratory of Materials Physics of \\ Ministry of Education of China, Zhengzhou University, Zhengzhou 450052, China \\ ${ }^{2}$ Department of Mathematics and Physics, Zhengzhou Institute of Aeronautical Industry \\ Management, Zhengzhou 450015, China \\ *E-mail: muk@zzu.edu.cn
}

\begin{abstract}
We numerically investigated the optical properties of planar nanorod complexes metamaterial that exhibits plasmonically induced transparency (PIT) effect. The interaction between two sides' nanorods and middle nanorod dimer leads to a single PIT band in transmission spectrum. Moreover, the double PIT windows can be realized by breaking the structure symmetry. The multi-bands PIT effect offers an excellent potential to manipulate the light speed in wide region.
\end{abstract}

Keywords: Plasmonic metamaterial; Plasmonically induced transparency; Slow light;

\section{Introduction}

Plasmonic nanostructures, supported collective electron excitions known as surface plasmon, have an ability to manipulate and concentrate light into deep-subwavelength scales. Although resistive heating losses in plasmonic metals severely limit the performance of optical devices, many successful applications have been achieved such as plasmonic lenses [1], sensors [2], perfect-absorbers [3], nanoantennas [4], switches [5], slow-light devices [6], surface-enhanced chips [7], and polarization controllers [8].

Recently, PIT phenomenon that can be analogized of the electromagnetically induced transparency (EIT) are experimentally demonstrated and investigated in plasmonic metamaterial. PIT can be used to manipulate light in metal nanostructures as an effective media with EIT-like optical properties, and can be realized in plasmonic metamaterial via destructive interference between the super-radiant bright mode and sub-radiant dark mode 
or by breaking the symmetry of the plasmonic metamaterial system. Zhang et al. [9] firstly predicted and investigated the Fano resonance and PIT effect in plasmonic structure with a dolmen-type slab arrangement, in which the metal strip functioned as an optical dipole antenna that served as a bright 'atom', and the two parallel metal strips with a small separation enabled the induction of anti-symmetric modes and acted as a dark 'atom'. Consequently, Liu et al. [10] experimentally observed PIT at Drude damping limit using stacked metamaterial. Up to date, a series of nanostructures have been taken into account to achieve the PIT effect, such as coupled resonators [11], optical antennas [12], waveguide systems [13], metallic nanoparticles array [14].

However, most of the PIT effects in plasmonic metamaterial have been observed at single wavelength. In this work, we theoretically discussed the optical properties of a designed planar metamaterial consisting of nanorod complexes. The interaction between two sides' nanorod and middle nanorod dimer brings to the destructive interference and PIT window in transmission spectrum. Contrast to the symmetrical nanorod complexes metamaterial that displays a single PIT wavelength, the asymmetrical structure achieved by adjusting coupling separation between the middle nanorod dimer and one side nanorod excite two detuned resonance modes, leading to double PIT windows.

\section{Designed metamaterial}

Fig. 1(a) and (b) show a typically periodical array of silver nanorod complexes planar metamaterial and a sketch of a unit cell with relevant geometric parameters, respectively. The designed metamaterial consists of four nanorods: a pair of parallel nanorods (termed as PPN) separated by an end-to-end nanorods dimer (termed as EEND). The original geometrical parameters of designed metamaterial are chosen as follows: $a=200 \mathrm{~nm}, b=100$ $\mathrm{nm}, d=30 \mathrm{~nm}, g_{1}=g_{2}=60 \mathrm{~nm}, w=40 \mathrm{~nm}$. The period of square lattice is set to $p=600 \mathrm{~nm}$ in $x$ and $y$ directions. The dielectric constants of silver measured by Palik [15] are used to model the planar metamaterial, and the thickness is fixed as $40 \mathrm{~nm}$. The nanorod complexes array is located on $\mathrm{SiO}_{2}$ substrate with dielectric constants $\varepsilon=2.07$. Numerical simulations were performed by the time domain solver of 3D electromagnetic package (CST Microwave Studio), where the computational domain was truncated by perfectly matched layers in the $z$-direction and the periodic boundary conditions were used to truncate the unit cell in the $x-y$ plane. During simulating, tetrahedron grids (thus a finite 
element method) were chosen and adaptive local mesh refinement was applied to create an improved mesh until the simulated results had sufficiently converged. The planar metamaterial was illuminated by a normal incident plane wave with an electric field polarization parallel to the $x$-axis.

\section{Numerical analysis and discussion}

EIT, which is a quantum interference effect in laser-driven atomic systems, gives rise to a sharp transparency window within a broad absorption spectrum. Compared with EIT behavior in atomic systems, PIT in plasmonic metamaterial has been observed and attracted enormous research interest due to its potential applications in novel photonics devices. Usually, the transparent window of PIT can be induced by accurately breaking the structural symmetry at the nanometer scale, which increases the difficulty to fabricate the PIT devices. In contrast, symmetric nanostructures can reduce the difficulty of preparation

\subsection{PIT in symmetric planar metamaterial with nanorod complexes}

Figure 2 (a) shows the simulated transmission spectra of the designed metamaterial consisting of four silver nanorods as described in figure 1. It is obvious that a narrow transparency window with $70 \%$ transmission appears at $297 \mathrm{THz}$ by exciting with $x$-polarized light (i.e. electric filed component parallel to the long axis of nanorods). The corresponding near field distribution of four nanorods metamaterial at transparency window is shown in figure 2(b). The field hot spots mainly locate at the ends of four nanorods, and the strongest electric field concentrates at the gap region of EEND due to the plasmon coupling. Furthermore, four nanorod complexes are excited simultaneously due to the resonance detuning, and the induced currents of PPN at both sides are in-phase and out-of-phase of $180^{\circ}$ with the middle EEND, as shown in figure 2(b) (green arrows), which implies destructive interference between the emitted fields from the two substructure arrays, leading to the PIT window in the designed metamaterial array.

In order to see metamaterial's inner workings of different sub-elements and their contributions to the PIT spectrum, we also independently calculate the transmitted spectra and filed pattern of PPN and EEND, respectively, as shown in figures 2(c)-(f). Individual PPN shown in insert in figure 2(b) exhibits a typical plasmon resonance of dipole oscillation at $285 \mathrm{THz}$, the positive and negative charges locate at the two ends of nanorods, respectively. In addition, the independent EEND array reveals a plasmon resonance at 309 
$\mathrm{THz}$, and the filed energy locates at the gap regions due to plasmonic coupling between nanoparticles in close proximity, as shown in figure 2(e) and (f).

In the spectral domain, the PIT coupling between two plasmon modes can be expressed with an analytical Fano interference model [16]:

$$
T(\omega)=T_{0}+a_{0} \omega+\sum_{i} \frac{2\left(f_{i}+q_{i}\right)^{2}}{1+4 f_{i}^{2}},\left(f_{i}=\frac{\omega-\omega_{i}}{\gamma_{i}}, i=1,2, \mathrm{~L}\right)
$$

where $T_{0}$ is constant background intensity, $\omega_{\mathrm{i}}$ and $\gamma_{\mathrm{i}}$ represent their resonance frequencies and line-widths, $q_{\mathrm{i}}$ are Fano asymmetrical coefficients. The additional linear term $a_{0} \omega$ is only concerned with the interface of two different dielectric materials. We fit the calculated transmission spectra (circles curves in figure 2(a), (c), and (e)) to equation (1) with different parameters, and the fitted results are shown as red curves in figure 2. Good agreement between the calculated spectra and the fitting curves with Fano model are realized.

\subsection{Slow light in nanorod complexes metamaterial}

The narrow band in the EIT window can be used to achieve strong dispersion and fabricate highly-confined slow light devices with a high group index. The controllable slow-light devices can manipulate photons for a long time inside the devices, which is powerful to enhance light-matter interactions. In general, the geometric parameters of sub-elements of metamaterial are critical in changing its optical characteristics.

Figure 3 shows the effect of the length of nanorods on the transmission spectrum and corresponding group index. In figure 3(a), the transmission spectra for different lengths of PPN and fixed rest parameters are investigated. Two distinct transmission bands are observed, and cross at one transmission resonance as $a=220 \mathrm{~nm}$. One of these bands is always centered at $1053 \mathrm{~nm}$, while the other one depends sensitively on $a$. The change of $a$ mainly influences the plasmon resonance position and width originated from PPN, whereas the plasmon mode from EEND is not affected greatly. The narrow profile in the PIT window is useful to achieve strong dispersion and confined slow-light device with a high group index. The controllable slow-light metamaterial can trap photons for a long time inside the structures, which is powerful to enhance light-matter interactions. The dispersion of transmission group index with different PPN length $a$ are represented in figure 3(b), and the corresponding group index $n_{\mathrm{g}}$ of metamaterial can be estimated by the following formula, 


$$
n_{g}=\frac{c_{0}}{v_{g}}=\frac{c_{0}}{D} \tau_{g}=-\frac{c}{D} \frac{d \phi(\omega)}{d \omega}
$$

Where $c_{0}$ is the light speed in free space, $v_{\mathrm{g}}$ and $\tau_{\mathrm{g}}$ are group velocity and delay time in metamaterial, respectively. D is spacing in the z-direction, and $\phi(\omega)$ is the transmission phase.

Figure 3(b) illustrates the corresponding group index map of figure 3(a). The large group indices appear around the crossing point at the transmission map due to the narrow transparency window and strong dispersion. Figure 3(c) shows the transmission spectra for different length of EEND and fixed rest parameters. Similar to figure 3(a), two crossing transmitted bands are observed in figure 3(c). One of these bands is always centered at 990 $\mathrm{nm}$, while the other one depends sensitively on $b$. Figure 3(d) shows the corresponding group index map with different length $b$.

\subsection{Double PIT in nanorod complexes metamaterial}

It is the interplay between electric dipole resonances that leads to the Fano resonance and PIT window. It is evident from figure 2 and the discussions above that two different electric dipoles with out-of-phase oscillations can be excited in the designed symmetrical metamaterial. Due the anti-parallel current distributions between PPN and EEND, the strong oscillating magnetic dipoles appear. However, the total magnetic moment of the whole structure approaches zero due to the cancellation effect in symmetrical structure. If the structure symmetry was broken, the net magnetic moment would be not equal to zero and the two magnetic dipoles would occur at different frequencies. Double PIT bands are expected by the interaction of the electric dipole resonance with magnetic dipoles at different frequencies. In order to validate this supposition, we calculate the modified metamaterial by breaking the structure symmetry.

Figure 4(a) and 4(b) show the transmission spectrum and corresponding group index of the asymmetrical metamaterial with the parameters $g_{1}=60 \mathrm{~nm}$ and $g_{2}=100 \mathrm{~nm}$, respectively. With the symmetry breaking, additional transparency window appears in transmission spectrum. The corresponding electric distributions at different frequencies (1, 2, 3, 4, and 5) are shown in left insert figures. It is shown that the additional transmission peak 4 originates from the interaction between two sides nanorods. The double PIT effect will induce two strong dispersion and slow light bands. Figure 4(b) shows the calculated group index 
spectrum, it is obvious that two frequency bands with large group index about 300 appear.

\section{Conclusions}

In conclusion, we proposed a planar plasmonic metamaterial with nanorod complexes arrangement. The interaction between the PPN and EEND leads to the PIT effect in transmission spectrum. Furthermore, the modified metamaterial by breaking the structure symmetry finally excite two adjacent PIT bands due to the introduction of magnetic momentum. The plasmonic metamaterial with multiple narrow PIT windows have extensional applications such as active plasmonic switching, slow-light optical devices, and narrow band filters.

\section{Acknowledgments}

This work was supported by the National Science Foundation of China (11504333, 61505178, 11404290, and 11404291), the research program of Henan province (142300410365, and 15A140014), and the Outstanding Young Talent Research Fund of Zhengzhou University (1521317007).

\section{References}

1) Y. Fu, and X. Zhou, Plasmonics 5(3), 287-310 (2010).

2) J. Wang, K. Mu, F. Ma, H. Zang, C. Fan, J. He, E. Liang, and P. Ding, Optics Communications 338, 399-405(2015).

3) J. Wang, C. Fan, P. Ding, J. He, Y. Cheng, W. Hu, G. Cai, E. Liang, and Q. Xue, Opt. Express 20(14), 14871-14878 (2012).

4) F. Bigourdan, J. P. Hugonin, F. Marquier, C. Sauvan, and J. J. Greffet, Phys. Rev. Lett. 116(10), 106803 (2016)

5) N. Jiang, L. Shao, and J. Wang, Adv. Mater. 26(20), 3282-3289 (2014)

6) J. Wang, B. Yuan, C. Fan, J. He, P. Ding, Q. Xue, and E. Liang, Opt. Express 21(21), 25159-25166 (2013).

7) J. Wang, X. Liu, L. Li, J. He, C. Fan, Y. Tian, P. Ding, D. Chen, Q. Xue and E. Liang, Journal of Optics 15(10): 105003 (2013).

8) J. Lin, J. B. Mueller, Q. Wang, G. Yuan, N. Antoniou, X. C. Yuan, and F. Capasso, Science 340(6130), 331-334 (2013).

9) S. Zhang, D. A. Genov, Y. Wang, M. Liu, and X. Zhang, Phys. Rev. Lett. 101(4), 047401 
(2008).

10) N. Liu, L. Langguth, T. Weiss, J. Kästel, M. Fleischhauer, T. Pfau, and H. Giessen, Nature materials, 8(9), 758-762 (2009).

11) W. Cao, R. Singh, C. Zhang, J. Han, M. Tonouchi, and W. Zhang, Appl. Phys. Lett., 103(10), 101106 (2013).

12) R. D. Kekatpure, E. S. Barnard, W. Cai, and M. L. Brongersma, Phys. Rev. Lett. 104(24), 243902 (2010).

13) X. Piao, S. Yu, and N. Park, Opt. Express, 20(17), 18994-18999 (2012).

14) W. Zhou, X. Wang, and J. Wang, Journal of Modern Optics, 62(12), 1027-1031 (2015).

15) E.D. Palik, Handbook of Optical Constants of Solids ,Academic Press, NewYork, 1985.

16) Y. Ma, Z. Li, Y. Yang, R. Huang, R. Singh, S. Zhang, J. Gu, Z. Tian, J. Han, and W. Zhang, Opt. Mater. Express, 1(3), 391-399 (2011) 


\section{Figure Captions}

Fig. 1. (a) Schematic of designed silver planar metamaterial with period $P$ (green: silver structure, gray: $\mathrm{SiO}_{2}$ substrate); (b) a unit cell with geometric scales $P=600 \mathrm{~nm}, a=200 \mathrm{~nm}$, $b=100 \mathrm{~nm}, d=30 \mathrm{~nm}, g_{1}=g_{2}=60 \mathrm{~nm}, w=40 \mathrm{~nm}$, and the thicknesses of all silver nanorods are $40 \mathrm{~nm}$.

Fig. 2. The simulated transmission spectrum ((a), (c), and (e)) and corresponding electric field distributions ((b), (d), and (f)) for designed planar metamaterial ((a) and (b)), individual PPN ((c) and (d)) and EEND ((e) and (f)), respectively.

Fig. 3. The transmission map ((a) and (b) and corresponding group index mapping ((b) and (d)) of designed planar metamaterial with different structural parameters $a$ and $b$, respectively.

Fig. 4. The transmission spectrum (a) and corresponding group index (b) of designed planar metamaterial with $g_{1}=60 \mathrm{~nm}$ and $g_{2}=100 \mathrm{~nm}$. The inset pictures show the electric field distributions at different frequencies. 


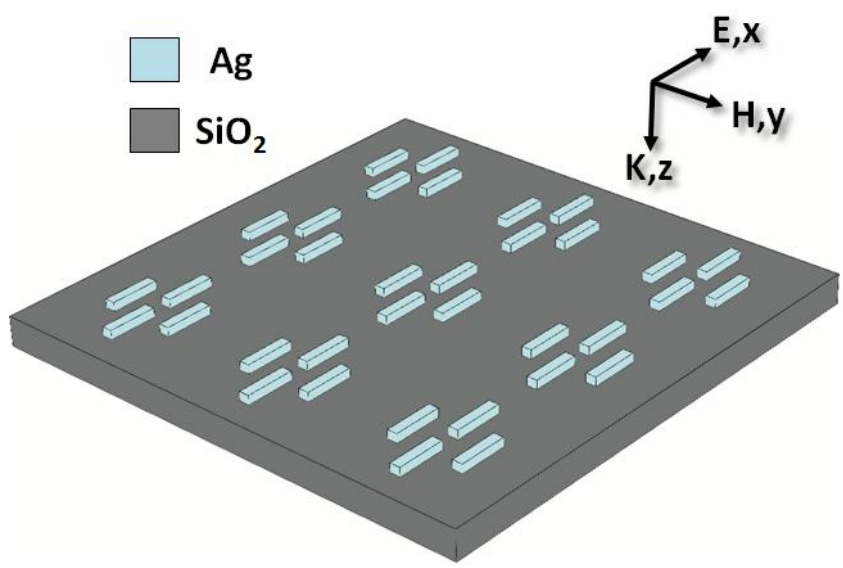

(a)

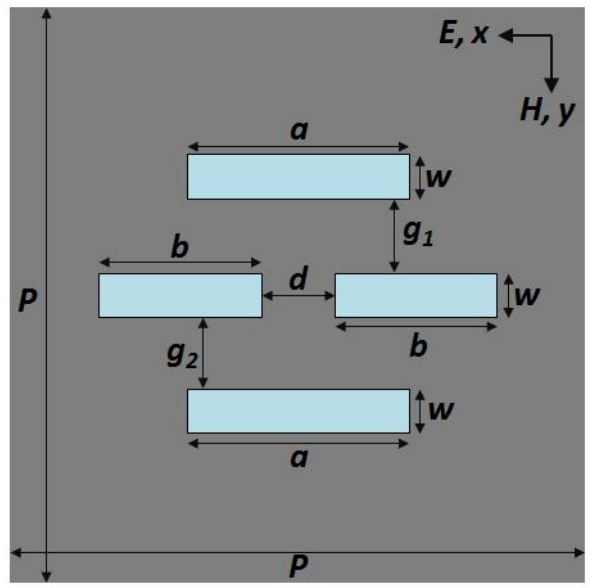

(b)

Fig.1 

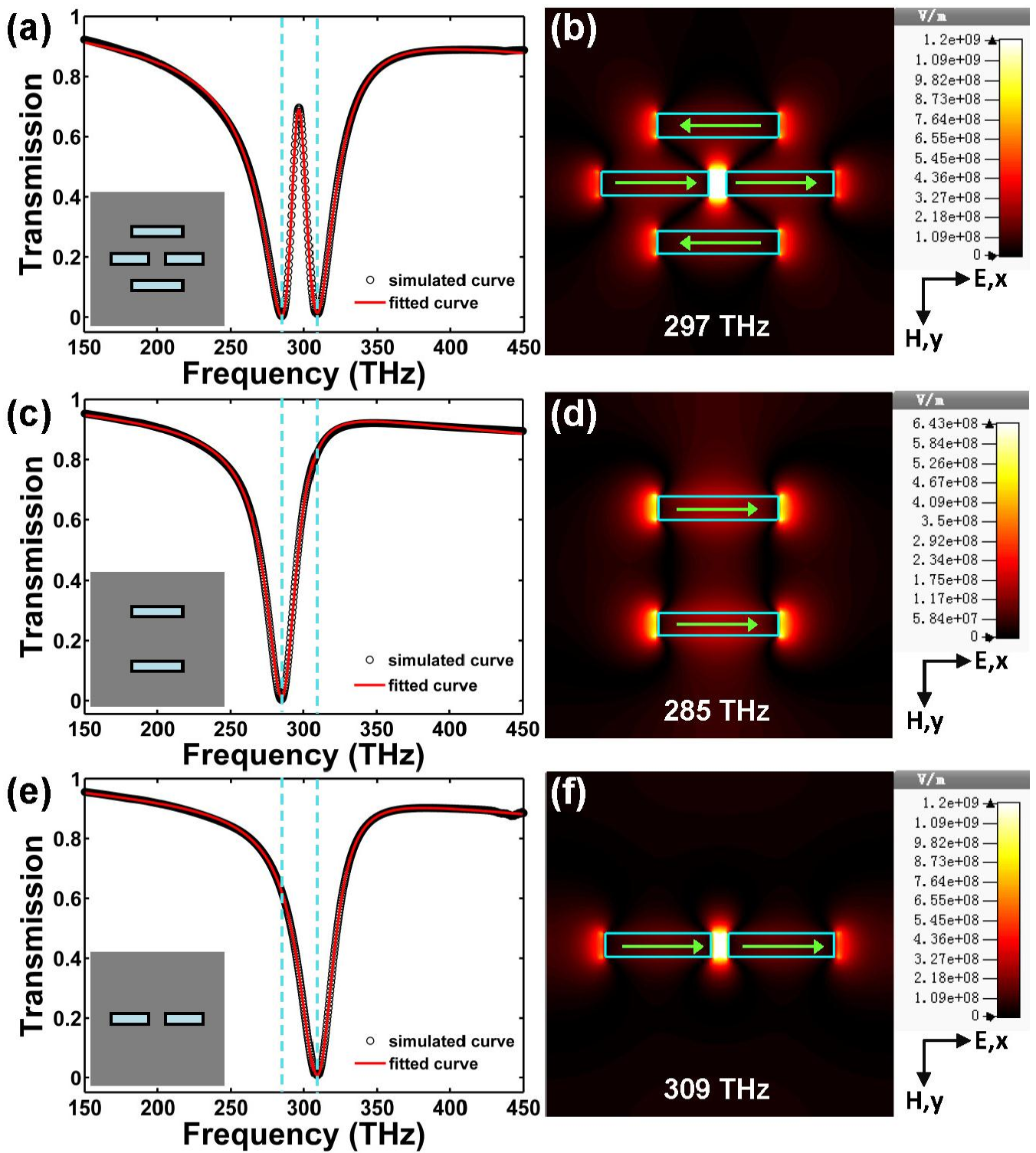

Fig. 2 


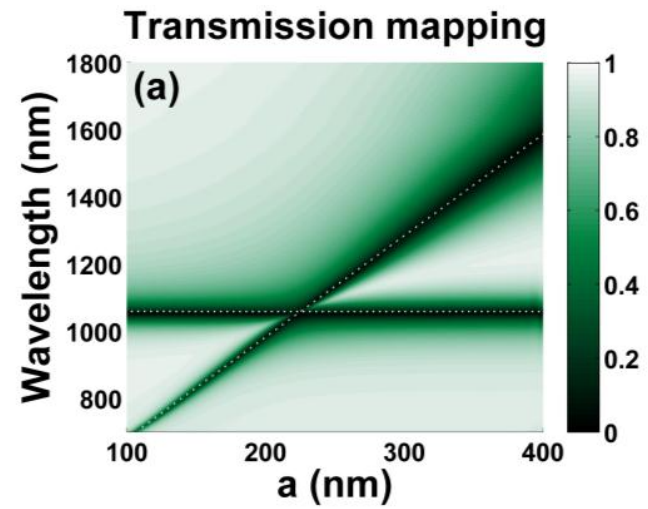

Transmission mapping

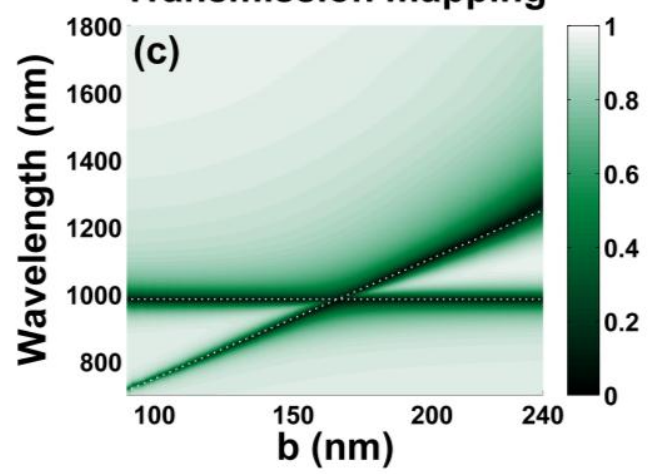

Fig. 3

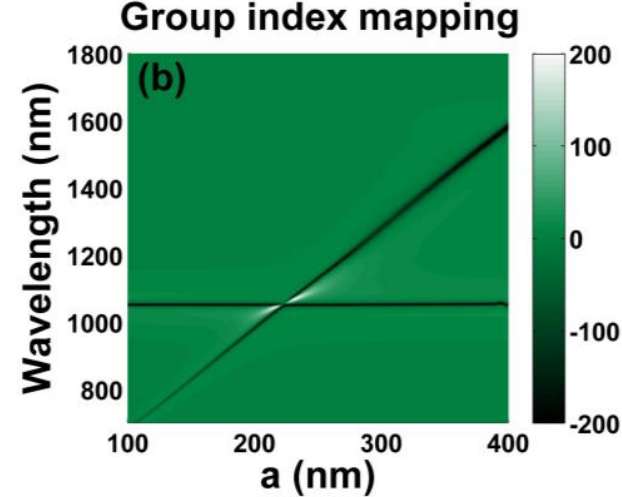

\section{Group index mapping}

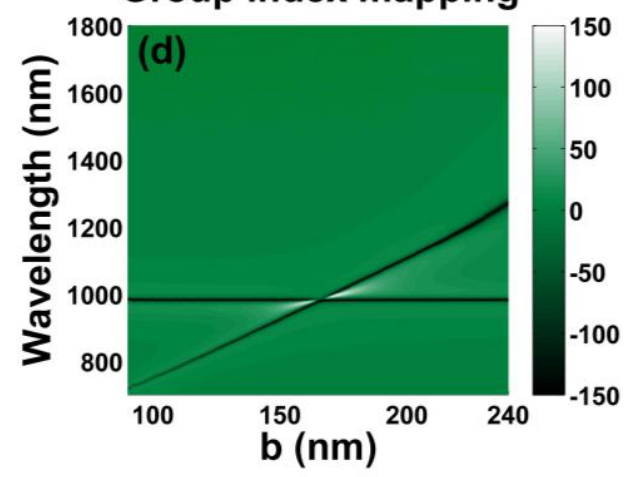




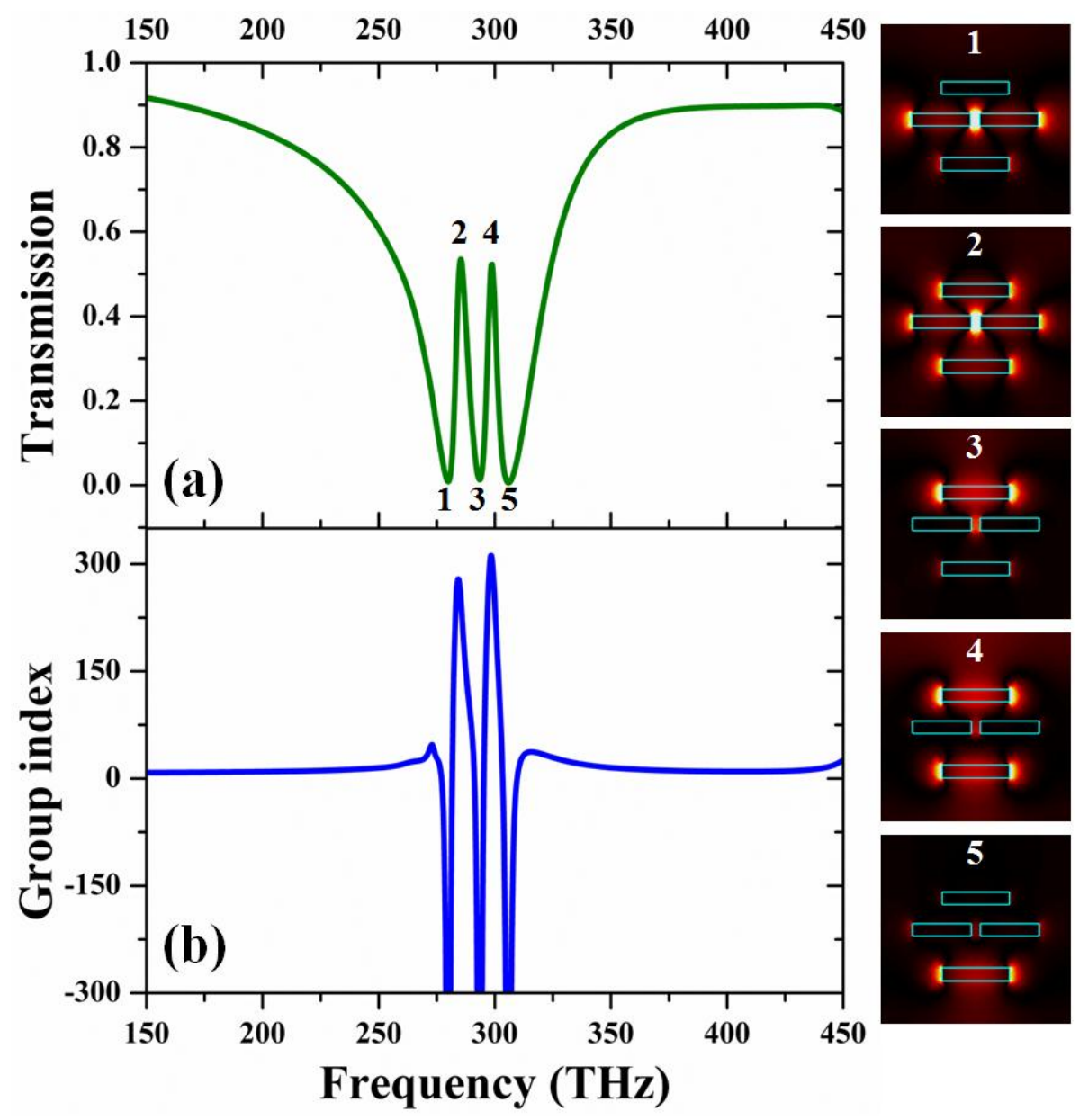

Fig. 4 